\title{
Sarcopenia is poor risk for unfavorable short- and long-term outcomes in stage I non-small cell lung cancer
}

\author{
Yusuke Takahashi ${ }^{1,2,3}$, Shigeki Suzuki ${ }^{1}$, Kenichi Hamada ${ }^{1}$, Takeo Nakada ${ }^{2}$, Yuko Oya ${ }^{2,4}$, Noriaki Sakakura ${ }^{2}$, \\ Hirokazu Matsushita ${ }^{3}$, Hiroaki Kuroda ${ }^{2}$ \\ ${ }^{1}$ Department of General Thoracic Surgery, Sagamihara Kyodo Hospital, Sagamihara, Kanagawa, Japan; ${ }^{2}$ Department of Thoracic Surgery, Aichi \\ Cancer Center Hospital, Nagoya, Aichi, Japan; ${ }^{3}$ Division of Translational Oncoimmunology, Aichi Cancer Center Research Institute, Nagoya, Aichi, \\ Japan; ${ }^{4}$ Department of Thoracic Oncology, Aichi Cancer Center Hospital, Nagoya, Japan \\ Contributions: (I) Conception and design: All authors; (II) Administrative support: All authors; (III) Provision of study materials or patients: Y \\ Takahashi, S Suzuki, K Hamada; (IV) Collection and assembly of data: Y Takahashi, S Suzuki, K Hamada; (V) Data analysis and interpretation: All \\ authors; (VI) Manuscript writing: All authors; (VII) Final approval of manuscript: All authors. \\ Correspondence to: Yusuke Takahashi, MD, PhD. Director, Department of Thoracic Surgery, Senior Researcher, Division of Translational \\ Oncoimmunology, 1-1 Kanoko-den, Chikusa-ku, Nagoya, Aichi 464-8681, Japan. Email: yusuketakahashigts@gmail.com.
}

\begin{abstract}
Background: Sarcopenia characterized by skeletal muscle loss may influence postoperative outcomes through physical decline and weakened immunity. We aimed to investigate clinical significance of sarcopenia in resected early-stage non-small cell lung cancer (NSCLC).

Methods: We retrospectively reviewed 315 consecutive patients with pathologic stage I NSCLC who had undergone lobectomy with systematic nodal dissection. Sarcopenia was defined as the lowest quartile of psoas muscle area on the 3rd vertebra on the high-resolution computed tomography (HRCT) image. Clinicopathological variables were used to investigate the correlation to postoperative complications as well as overall and recurrence-free survival.

Results: Upon multivariable analysis, male sex [odds ratio $(\mathrm{OR})=5.780,95 \%$ confidence interval (CI): 2.681-12.500, $\mathrm{P}<0.001$ ], and sarcopenia ( $\mathrm{OR}=21.00$, 95\% CI: $10.30-42.80, \mathrm{P}<0.001$ ) were independently associated with postoperative complications. The sarcopenia group showed significantly lower 5-over all survival $(84.4 \%$ vs. $69.1 \%, \mathrm{P}<0.001)$ and recurrence-free survival $(77.2 \%$ vs. $62.0 \%, \mathrm{P}<0.001)$ comparing with the non-sarcopenia group. In a multivariable analysis, sarcopenia was an independent prognostic factor [hazard ratio $(\mathrm{HR})=1.978,95 \% \mathrm{CI}: 1.177-3.326, \mathrm{P}=0.010$ ] together with age $\geq 70$ years $(\mathrm{HR}=1.956,95 \%$ CI: 1.141-3.351, P=0.015) and non-adenocarcinoma histology (HR =1.958, 95\% CI: 1.159-3.301, P=0.016).

Conclusions: This is the first study which demonstrates that preoperative sarcopenia is significantly associated with unfavorable postoperative complications as well as long-term survival in pathologic stage I NSCLC. This readily available factor on HRCT may provide valuable information to consider possible choice of surgical procedure and perioperative management.
\end{abstract}

Keywords: Skeletal muscle mass; psoas muscle; anti-cancer immunity; node-negative; surgery

Submitted May 31, 2020. Accepted for publication Oct 30, 2020.

doi: $10.21037 / \mathrm{atm}-20-4380$

View this article at: http://dx.doi.org/10.21037/atm-20-4380

\section{Introduction}

Non-small cell lung cancer (NSCLC) is one of the most common cause of cancer-deaths worldwide (1). Lobectomy with systematic lymph node dissection has been a standard therapeutic option for early-stage NSCLC $(2,3)$. A major lung resection including lobectomy often causes impaired immune system as well as cardiopulmonary function, resulting in increased risk of postoperative complications $(4,5)$. Despite recent advances of diagnostics and 
therapeutics in NSCLC, approximately $20-30 \%$ of patients with completely resected early-stage NSCLC develop recurrence which may arise from occult micrometastasis facilitated by tumor-promoting microenvironment during perioperative period (6), while post-recurrence survival is still poor (7).

Preoperative clinical status including age, tumor stage, and smoking history is generally assessed in patients with early-stage NSCLC. A better prediction of prognosis with using pretreatment clinical factors in early-stage NSCLC may lead appropriate decision-making on therapeutic strategy.

Recently, frailty is most often defined as a state of physiological decline associated with ageing $(8,9)$. It was characterized by unintentional weight loss, weakness, exhaustion, slow walking speed, and low physical and mental activity so that frailty can be evaluated with using various subjective methods, for instance, selfevaluation questionnaires and the assessment of certain physical activities of daily life (10). Since sarcopenia does more specifically focus on decline of physical activity characterized by loss of skeletal muscle (11), sarcopenia is more quantitative and suitable for preoperative evaluation (12).

Lots of publications demonstrated its association to postoperative survival outcome in various pathologies including cardiovascular $(13,14)$, chronic renal failure (15), and sepsis (16). In addition, its clinical significance in predicting survival outcomes of solid malignancies has been well documented (17-20). However, the clinical significance of sarcopenia in patients undergoing lung resection has not been well understood to date. Especially, prognostic significance of sarcopenia in thoracic surgery for NSCLC is controversial. On the background, we hypothesized that sarcopenia is associated with higher risk of either short- and long-term outcomes in patients with NSCLC resected. The aim of this study was to clarify whether sarcopenia is significant in predicting postoperative complications and long-term survival in patients with pathologic stage I NSCLC. We present the following article in accordance with the REMARK reporting checklist (available at http://dx.doi.org/10.21037/atm-20-4380).

\section{Methods}

\section{Study design and patient's selection}

This retrospective study was approved by the institutional review board at Sagamihara Kyodo Hospital (2018-004) and performed in adherence with the Declaration of Helsinki (as revised in 2013). During the period from April 2007 to July 2015, a total of 490 consecutive patients underwent surgery for primary lung cancer at our institution. Medical records of the patients were reviewed and the patients with pathologic stage I NSCLC who underwent complete resection were included as previously described $(21,22)$. The following exclusion criteria were applied: (I) patients with history of lung cancer within past two years; (II) those with clinical stage II-IV disease; (III) those had undergone sublobar resection; (IV) those had undergone induction therapy prior to surgery, and (V) those with carcinoid or large cell neuroendocrine carcinoma.

\section{Preoperative examination and surgical treatment}

Preoperatively, a physical examination, blood chemistry analysis, pulmonary function test, bronchoscopy, chest radiography, and computed tomography (CT), brain magnetic resonance imaging (MRI), and integrated positron emission tomography scan and CT scan (PET/CT) were routinely performed. All patients underwent a lobectomy with systematic lymph node dissection. Our operative indication of lobectomy is predicted postoperative forced expiratory volume in 1 second (FEV1) larger than $0.8 \mathrm{~L}$.

\section{Preoperative CT scan and evaluation of sarcopenia}

CT scan was routinely performed using 64-detector-row CT scanner (Light Speed VCT; General Electric, CT, USA). Whole chest and abdomen were scanned during a breathhold at deep inspiration phase in supine position, and $1.25-\mathrm{mm}$ thick high-resolution images were reconstructed using standard spatial-frequency reconstruction algorithm. Digital imaging and communications in medicine data was transferred to the commercially available workstation as previously described (Synapse Vincent; Fujifilm Medical Co., Tokyo, Japan) (23). On the workstation, psoas muscle area, a validated marker of sarcopenia, at the level of the top of the iliac crest was semi-automatically measured in all patients as shown in Figure 1. Sarcopenia was defined as the lowest sex-specific quartile in the average of both psoas muscle areas at preoperative CT scan as previously described $(13,24)$.

\section{Definition of postoperative complications}

Postoperative complications were evaluated in accordance 


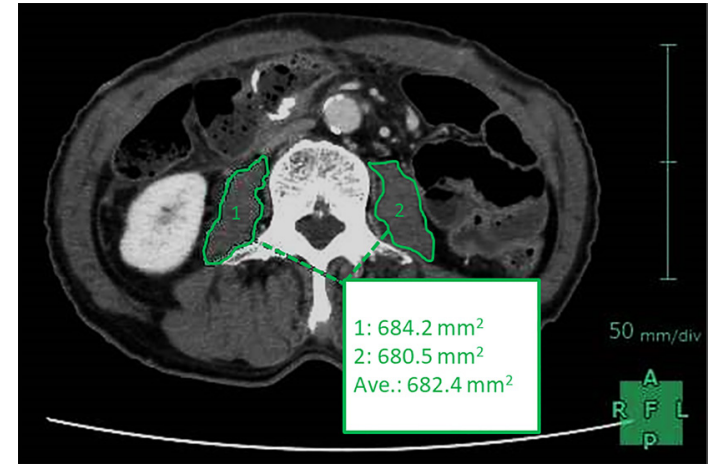

Figure 1 Representative high-resolution computed tomography image used in measuring psoas muscle area.

with Common Terminology Criteria for Adverse Events (version 4.03) $(4,25)$. Severe morbidities were defined as grade 3 and higher of the classification, occurring within 30 days after surgery $(3,5,25)$.

Based on our postoperative follow-up policy, we examined patients at 3-month intervals for the first 3 years and typically at 6-month intervals thereafter on an outpatient basis in accordance with the National Comprehensive Cancer Network guidelines $(21,26)$. The routine follow-up evaluation included physical examination, chest radiography, chest CT, and blood analysis including serum tumor markers. Whenever any symptoms or signs of recurrence were detected, further evaluation was performed, including CT of the chest and abdomen, brain MRI, and bone scintigraphy, or PET/CT. Biopsy was performed for histological confirmation for diagnosis of recurrent disease if necessary. If unfeasible, radiological evidence of recurrent lesion was accepted by the institutional multidisciplinary board of lung cancer.

\section{Statistical analysis}

Overall survival (OS) was measured by comparing the date of surgery to the date of death from any cause or the date on which the patient was last known to be alive. The data cut-off was December 2019. Survival curves were plotted according to the Kaplan-Meier method and comparisons were drawn using the log-rank test in a univariable analysis. The recurrence-free survival (RFS) time was measured as the interval between the date of surgery and the date of recurrence, the date of death from any cause, or the most recent date on which the patient was last known to be alive. To determine the independent prognostic factors, a multivariable analysis was conducted using the Cox proportional hazard model as previously described $(22,27)$. Two-category comparisons were performed using the Pearson $\chi^{2}$ test and the Fisher exact test for quantitative data. We used the following variables into the statistical models: age at surgery (years), smoking index (pack-years), body mass index $\left(\mathrm{kg} / \mathrm{m}^{2}\right)$, sex, diabetes mellitus, sarcopenia, forced vital capacity (FVC; L), \%FVC, FEV1 (L), FEV1/ FVC (\%), and tumor diameter on high-resolution computed tomography (HRCT) $(\mathrm{cm})$. All the tests were two-sided, and $P$ values less than 0.05 were considered statistically significant. R software (version 3.2.2; R Foundation for Statistical Computing, Vienna, Austria) was used for the statistical analysis.

\section{Results}

\section{Baseline demographic data}

We included 489 patients with resected and pathologically diagnosed NSCLC patients. Then, 115 patients who had undergone sublobar resections, 3 with prior history of lung cancer, 2 who had undergone induction therapy before lung resection, 54 with clinically diagnosed stage II-IV disease, and 3 patients with carcinoid as well as 4 patients with large cell neuroendocrine carcinoma were excluded from the primary cohort in accordance with the exclusion criteria above (Figure 2). Our final study cohort was 315 patients with NSCLC who had undergone lobectomy with systematic lymph node dissection. The study cohort included $192(61.0 \%)$ men and $123(39.0 \%)$ women, with a median age of 70.0 years [quartile deviation (QD): 6.0 years]. Of the 315 patients, 201 (63.8\%) had smoking history (Table 1). The median follow-up time was 58.8 months (range, 0.7-137.0 months). Histologically, 229 (72.7\%) had adenocarcinoma, 73 (23.2\%) had squamous cell carcinoma, and 13 (4.1\%) had other histology. EGFR mutation status was wild type in $160(50.8 \%)$, mutated in $95(30.2 \%)$, and unknown in 60 (19.0\%). For each patient, psoas muscle area was semi-automatically measured on preoperative HRCT as above (Figure 1). A median psoas muscle area was $673.53(\mathrm{QD}: 166.12) \mathrm{mm}^{2}$ for men and $525.52(\mathrm{QD}: 75.771) \mathrm{mm}^{2}$ for women $(\mathrm{P}<0.001)$ and the distribution was shown in Figure S1. In the current study, sarcopenia was defined as the lowest sex-specific quartile in the average of both psoas muscle areas at preoperative HRCT scan as previously described (13). 


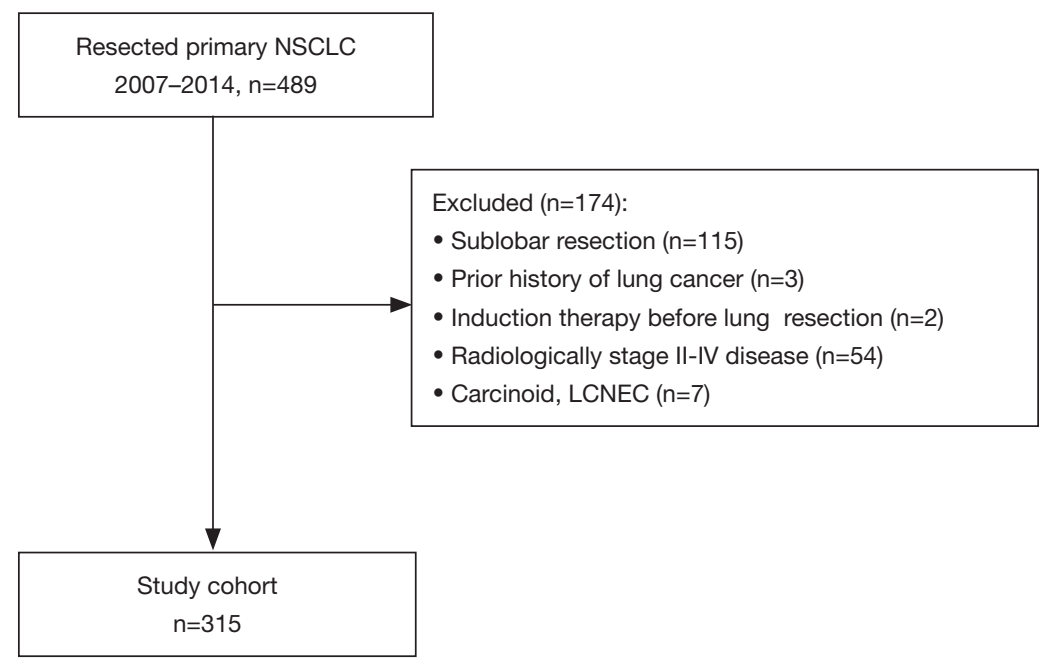

Figure 2 CONSORT diagram which demonstrates patient number included and excluded (the numbers include overlapping).

We thus performed following analysis with the cut-off value of $491.53 \mathrm{~mm}^{2}$ for men and $460.55 \mathrm{~mm}^{2}$ for women. Consequently, we have observed 38 cancer-related and 29 non-cancer-related deaths during the follow-up period in this study. Also, 54 patients have experienced recurrence.

\section{Comparison of clinical data and sarcopenia as well as postoperative complications}

Table 2 demonstrates correlation between sarcopenia and preoperative variables. The variables other than FEV1 $(\mathrm{P}<0.001)$ did not significantly differ according to presence or absence of sarcopenia.

Next, we investigated correlation between postoperative complications and clinical factors. As shown in Table 3, larger smoking index $(\mathrm{P}<0.001)$, lower body mass index $(\mathrm{P}=0.027)$, male sex $(\mathrm{P}<0.001)$, and sarcopenia $(\mathrm{P}<0.001)$ were significantly associated with postoperative severe complications. Among the postoperative severe complications observed in 82 cases $(26.0 \%)$, pulmonary fistula $(41 / 315,13.0 \%)$ was the most frequent, followed by atrial fibrillation (18/315, 5.7\%), atelectasis (15/315, 4.8\%), and pneumonia $(14 / 315,4.4 \%)$ as shown in Table S1. To investigate independent risk factors, a multivariable logistic regression analysis was performed (Table 4). As a result, male sex [odds ratio $(\mathrm{OR})=5.780 ; 95 \%$ confidence interval $(\mathrm{CI})$, 2.681-12.500; $\mathrm{P}<0.001]$ and sarcopenia $(\mathrm{OR}=21.00 ; 95 \%$ CI, 10.30-42.80; $\mathrm{P}<0.001)$ were independently associated with postoperative severe complications.

\section{Survival analyses}

We then performed univariable and multivariable prognostic analysis of OS using Cox hazard model (Table 5). In the univariable analysis, age at surgery $\geq 70$ years [hazard ratio $(\mathrm{HR})=2.027 ; 95 \% \mathrm{CI}, 1.217-3.376 ; \mathrm{P}<0.001]$, male sex $(\mathrm{HR}=2.387 ;$ 95\% CI, 1.359-4.184, $\mathrm{P}<0.001)$, smoking index $\geq 400$ pack-years ( $\mathrm{HR}=2.496 ; 95 \%$ CI, 1.467-4.247, $\mathrm{P}<0.001$ ), serum carcinoembryonic antigen (CEA) $\geq 5.0 \mathrm{ng} / \mathrm{mL}(\mathrm{HR}=1.991 ; 95 \% \mathrm{CI}, 1.183-3.350, \mathrm{P}<0.001)$, sarcopenia ( $\mathrm{HR}=2.237$; 95\% CI, 1.374-3.625, $\mathrm{P}<0.001$ ), and non-adenocarcinoma histology $(\mathrm{HR}=2.771 ; 95 \%$ CI, 1.708-4.464, $\mathrm{P}<0.001$ ) were significant unfavorable prognostic factors in OS. Then, multivariable analysis using these factors revealed that age at surgery $\geq 70$ years $(\mathrm{HR}=1.956 ; 95 \% \mathrm{CI}, 1.141-3.351, \mathrm{P}=0.015)$, serum CEA $\geq 5.0 \mathrm{ng} / \mathrm{mL} \quad(\mathrm{HR}=1.040 ; 95 \%$ CI, 1.024-1.056, $\mathrm{P}<0.001$ ), sarcopenia (HR $=1.978$; 95\% CI, 1.177-3.326, $\mathrm{P}=0.010)$, and non-adenocarcinoma $(\mathrm{HR}=1.958 ; 95 \%$ CI, 1.159-3.301, $\mathrm{P}=0.016$ ) were independently associated with unfavorable OS. Additionally, prognosticators of RFS were investigated using Cox regression models. Univariable analysis demonstrated that age at surgery $\geq 70$ years $(\mathrm{HR}=1.593 ; 95 \% \mathrm{CI}, 1.051-2.415, \mathrm{P}=0.022)$, male sex $(\mathrm{HR}=1.542 ; 95 \% \mathrm{CI}, 1.009-2.325, \mathrm{P}=0.043)$, smoking index $\geq 400$ pack-years (HR $=1.567 ; 95 \% \mathrm{CI}$, $1.034-2.376, \mathrm{P}=0.034)$, serum CEA $\geq 5.0 \mathrm{ng} / \mathrm{mL}$ (HR $=1.034 ; 95 \%$ CI, $1.021-1.049, \mathrm{P}<0.001)$, and sarcopenia (HR $=2.316 ; 95 \%$ CI, $1.592-3.324, \mathrm{P}<0.001)$ were significant prognostic factors in RFS. In the multivariable analysis, age 
Table 1 Baseline characteristics of the patient cohort $(n=315)$

\begin{tabular}{|c|c|}
\hline Characteristics & Value \\
\hline Age, median [range] & 70 [35-88] \\
\hline \multicolumn{2}{|l|}{ Gender } \\
\hline Male & 192 \\
\hline Female & 123 \\
\hline \multicolumn{2}{|l|}{ Smoking history } \\
\hline Never-smoker & 114 \\
\hline Ex or current smoker & 201 \\
\hline Body mass index $\left(\mathrm{kg} / \mathrm{m}^{2}\right)$, median (range) & $22.5(14.9-33.3)$ \\
\hline FVC (L), median (range) & $3.04(1.44-5.99)$ \\
\hline \%FVC (\%), median (range) & $109.2(53.1-174.7)$ \\
\hline FEV1 (L), median (range) & $2.19(1.14-4.76)$ \\
\hline FEV1/FVC (\%), median (range) & $74.1(35.7-98.9)$ \\
\hline \multicolumn{2}{|l|}{ Comorbidities } \\
\hline Hypertension & 100 \\
\hline Chronic obstructive pulmonary disease & 55 \\
\hline Diabetes mellitus & 47 \\
\hline Interstitial lung disease & 12 \\
\hline Infectious lung disease & 8 \\
\hline Tuberculosis & 6 \\
\hline Acute coronary disease & 8 \\
\hline Chronic kidney disease & 3 \\
\hline \multicolumn{2}{|l|}{ Histology } \\
\hline Adenocarcinoma & 229 \\
\hline Squamous cell carcinoma & 73 \\
\hline Adenosquamous carcinoma & 5 \\
\hline Large cell carcinoma & 8 \\
\hline
\end{tabular}

FVC, forced vital capacity, FEV1, forced expiratory volume in 1 second.

at surgery $\geq 70$ years $(\mathrm{HR}=1.594 ; 95 \%$ CI, $1.065-2.319$, $\mathrm{P}=0.034)$, serum $\mathrm{CEA} \geq 5.0 \mathrm{ng} / \mathrm{mL}(\mathrm{HR}=1.037 ; 95 \% \mathrm{CI}$, 1.021-1.056, $\mathrm{P}<0.001)$, and sarcopenia $(\mathrm{HR}=1.914 ; 95 \%$ CI, 1.237-2.894, $\mathrm{P}=0.005)$ were independent prognostic factors of RFS (Table S2).

To illustrate survival differences according to presence or absence of sarcopenia, Kaplan-Meier curves were generated. As shown in Figure 3A, non-sarcopenia group had a 5-year OS probability of $84.4 \%$ which was significantly better compared with $69.1 \%$ of sarcopenia group $(\mathrm{P}<0.001)$. Sarcopenia was also associated with unfavorable RFS (5-year RFS, $62.0 \%$ of sarcopenia group vs. $77.2 \%$ of nonsarcopenia group; $\mathrm{P}<0.001$; Figure $3 B$ ).

\section{Discussion}

The current data demonstrated that sarcopenia was an independent risk factor for postoperative severe complications as well as RFS in patients with resected stage I NSCLC. Our hypothesis was shown to be correct as above.

Our findings may be reliable as our study cohort which was consisted of homogeneous patients in terms of disease stage and race. Several previous reports have demonstrated that sarcopenia is a prognostic factor after surgery in resected NSCLC $(28,29)$. To our knowledge, only 3 publications documented significant correlation between sarcopenia and postoperative complication after surgery NSCLC (30-32). On the other hand, it has been well documented regarding significant correlation between sarcopenia and postoperative complications following surgical resection in other solid malignancy (33-38). In NSCLC patients, however, there are some reports which demonstrated sarcopenia as an unfavorable prognostic impact on OS (29,32,39-43), among them 3 employed only stage I NSCLC patients $(40,41,43)$. Altogether, this is the first publication which demonstrates clinical significance of sarcopenia on both long- and short-term outcomes after surgery in patients with stage I NSCLC. The findings are mostly consistent with previous literature as above (29-32,39-43), even our data is limited to stage I NSCLC which may emphasize clinical significance of sarcopenia in surgically resected NSCLC. Previous studies used various measurement methods and cut-off values in evaluating sarcopenia. Although our "sarcopenia group" may reflect slightly weakened physical properties because we employed the lowest quartile of psoas muscle area as the cut-off value, it should include some of just relatively sarcopenic patients in the current study. Although there are some controversies respect to evaluation methods of sarcopenia, our findings may suggest clinical relevance in short- and long-term outcomes with evaluating "pre-sarcopenia" even in earlystage NSCLC.

Skeletal muscle loss is emerging issue in the context of sarcopenia and frailty which was reported to be decreasing as a patient becomes older with the decreases at an annual rate of $1 \%$ to $2 \%$ in average (44). Whereas the current data 
Table 2 Baseline patient characteristics according to presence or absence of sarcopenia

\begin{tabular}{lccc}
\hline Factors & Non-sarcopenia $(\mathrm{n}=236)$ & Sarcopenia $(\mathrm{n}=79)$ & P value* \\
\hline Age at surgery (years) & $70.2 \pm 7.5$ & $70.4 \pm 5.0$ & 0.197 \\
Smoking habit (pack-years) & $410 \pm 480$ & $720 \pm 520$ & 0.468 \\
Body mass index $\left(\mathrm{kg} / \mathrm{m}^{2}\right)$ & $22.60 \pm 1.75$ & $22.20 \pm 2.07$ & 0.144 \\
Male (\%) & $142(60.2)$ & $46(58.2)$ & 1.000 \\
Diabetes mellitus (\%) & $24(10.2)$ & $12(15.2)$ & 0.352 \\
FVC (L) & $3.10 \pm 0.59$ & $2.93 \pm 0.52$ & 0.064 \\
\%FVC & $110.0 \pm 11.8$ & $107.0 \pm 12.7$ & 0.069 \\
FEV1 (L) & $2.32 \pm 0.50$ & $1.97 \pm 0.28$ & $<0.001$ \\
FEV1/FVC (\%) & $74.60 \pm 6.43$ & $72.90 \pm 6.50$ & 0.160 \\
Tumor diameter on HRCT $(\mathrm{cm})$ & $2.50 \pm 0.84$ & $2.50 \pm 0.68$ & 0.822 \\
\hline
\end{tabular}

*, Mann-Whitney U-test for non-categorical factors and Fischer's exact test for categorical factors. FVC, forced vital capacity; FEV1, forced expiratory volume in 1 second; HRCT, high resolution computed tomography.

Table 3 Baseline patient characteristics according to presence or absence of postoperative complications

\begin{tabular}{lccc}
\hline Factors & Complication $(\mathrm{n}=82)$ & No complication $(\mathrm{n}=233)$ & $\mathrm{P}$ value* \\
\hline Age at surgery (years) & $71.2 \pm 5.3$ & $70.3 \pm 6.5$ & 0.242 \\
Smoking index (pack-years) & $920 \pm 475$ & $300 \pm 450$ & $<0.001$ \\
Body mass index $\left(\mathrm{kg} / \mathrm{m}^{2}\right)$ & $21.90 \pm 1.95$ & $22.70 \pm 1.76$ & 0.027 \\
Male (\%) & $65(79.3)$ & $127(54.3)$ & $<0.001$ \\
Diabetes mellitus (\%) & $12(14.6)$ & $26(11.1)$ & 0.453 \\
Sarcopenia & $53(64.6)$ & $26(11.1)$ & $<0.001$ \\
FVC (L) & $3.23 \pm 0.58$ & $2.99 \pm 0.53$ & 0.057 \\
\%FVC & $106.0 \pm 13.4$ & $110.0 \pm 11.8$ & 0.189 \\
FEV1 (L) & $2.07 \pm 0.55$ & $2.21 \pm 0.49$ & 0.899 \\
FEV1/FVC $(\%)$ & $72.10 \pm 9.88$ & $74.60 \pm 5.78$ & 0.059 \\
Tumor diameter on HRCT (cm) & $2.51 \pm 0.60$ & $2.46 \pm 0.72$ & 0.117 \\
\hline
\end{tabular}

*, Mann-Whitney U-test for non-categorical factors and Fischer's exact test for categorical factors. FVC, forced vital capacity; FEV1, forced expiratory volume in 1 second; HRCT, high resolution computed tomography.

suggests that age at surgery is not necessarily related to the presence or absence of sarcopenia, which may be supported by the previous publications $(13,30,43)$. It is thus indicated that sarcopenia may be caused by genetic factors, exercise habit, and nutrition status in addition to ageing.

Possible explanations for unfavorable long-term prognostic impact of sarcopenia are the following. First, sarcopenia is associated with impaired antitumor immunity via increased transforming growth factor $\beta$ (TGF- $\beta$ ) and interleukin (IL)-6 in previously reported data $(45,46)$. It may lead higher risk of recurrence and cancer-related death, resulting in shorter RFS and OS of sarcopenic patients. Second, it is reported that sarcopenic state could lead higher risk of short- and long-term complications following 
Table 4 Multivariable logistic regression analysis of risk factors for postoperative complications

\begin{tabular}{lccc}
\hline Variables & Odds ratio & $95 \% \mathrm{Cl}$ & $\mathrm{P}$ value* \\
\hline Smoking index $\geq 400$ pack-years (vs. $<400)$ & 1.020 & $0.450-2.300$ & 0.968 \\
Body mass index $<18.5$ (vs. $\geq 18.5)$ & 1.536 & $0.654-3.597$ & 0.324 \\
Male sex (vs. female) & 5.780 & $2.681-12.500$ & $<0.001$ \\
Sarcopenia (vs. non-sarcopenia) & 21.00 & $10.30-42.80$ & $<0.001$ \\
FEV1/FVC $<70.0$ (vs. $\geq 70.0)$ & 1.610 & $0.817-3.170$ & 0.169 \\
\hline
\end{tabular}

*, logistic regression analysis was used. Cl, confidence interval; FVC, forced vital capacity; FEV1, forced expiratory volume in 1 second.

Table 5 Univariable and multivariable Cox-regression analysis of overall survival

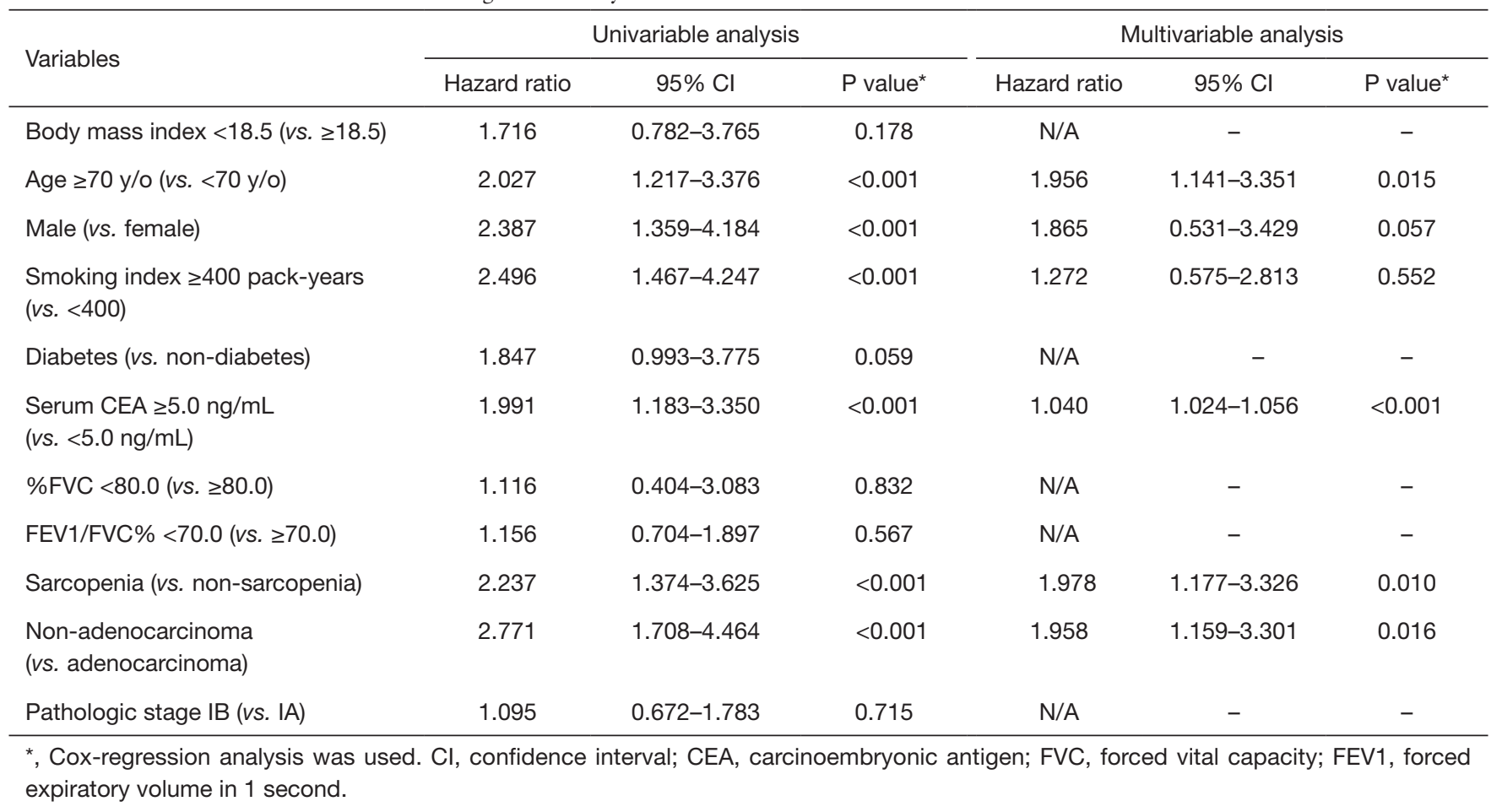

surgical resection, including pneumonia which may result in higher risk of non-cancer related death $(47,48)$. Muscle loss and dysfunction cause impaired respiratory function with increased TGF- $\beta$, IL- 6 , and tumor necrosis factor- $\alpha$ $(\mathrm{TNF}-\alpha)$ (49-52). Third, sarcopenic patients are likely to be more sensitive to chemotherapy toxicity and have a lower response to chemotherapy $(53,54)$.

Particularly, it is notable that there is certain correlation between sarcopenia and inflammation as well as anticancer immunity. However, the actual detailed pathophysiology of sarcopenia is complex and still unclear to date (55). Underlying cellular mechanisms of both sarcopenia and frailty are not well understood. Sarcopenic patients are reportedly on the systemic low-grade proinflammatory state in which increased pro-inflammatory cytokines including IL-1 $\beta$, IL-6, TNF- $\alpha(56,57)$ and decreased IL-10 secreted by regulatory $\mathrm{T}$ lymphocyte was demonstrated in some publications (58). On the flip side, adiposity leads increased secretion of pro-inflammatory adipokines including lectin and decreased anti-inflammatory adipokines including adiponectin (59). In addition, reduced physical activity with ageing reportedly increases proinflammatory cytokines (60) and increased serum IL-6 is also significantly associated with development of sarcopenia (61). Inflammatory cytokines, 
A

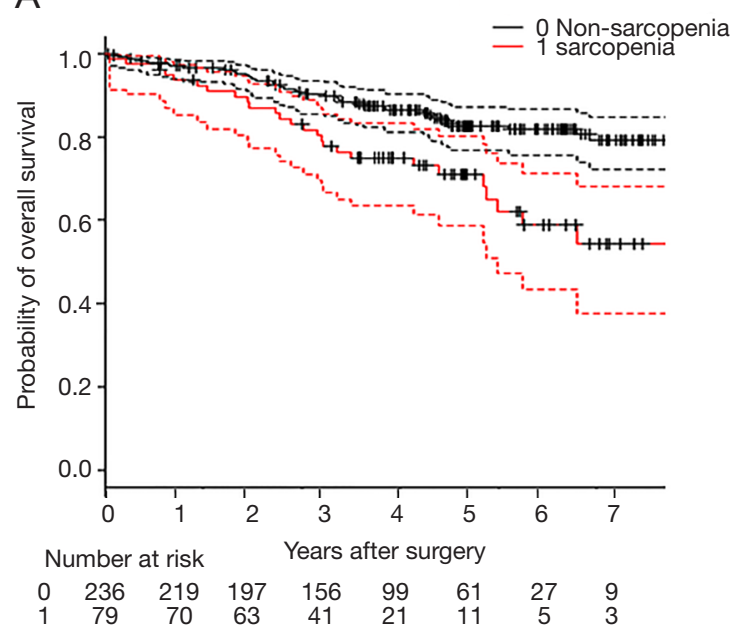

B

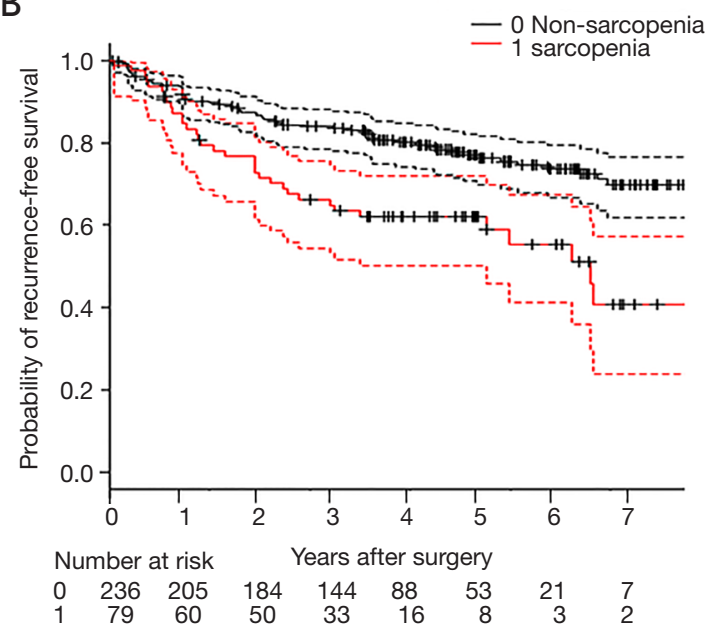

Figure 3 Overall and recurrence-free survival curves are well stratified according to sarcopenia. (A) Kaplan-Meier overall survival curves for patients with stage I NSCLC of sarcopenia (sarcopenia 1) and non-sarcopenia (sarcopenia 0) groups. The dotted curves represent 95\% CI curves of the groups. (B) Kaplan-Meier recurrence-free survival curves for patients with stage I NSCLC of sarcopenia (sarcopenia 1) and non-sarcopenia (sarcopenia 0) groups. The dotted curves represent 95\% CI curves of the groups. NSCLC, non-small cell lung cancer; CI, confidence interval.

which was produced by skeletal muscle after exercise, play a crucial role in complexed immune system (62). Then, inflammation with increased neutrophil migration causes muscle damage and impaired anti-tumor immune response (63).

Possible clinical application is another point. An important application of sarcopenia is potential to stratify patients at risk for recurrence as well as death. Also, there are some considerable choices in modifying perioperative management and treatment in sarcopenic patients. First, sarcopenic patients were at higher risk for short- and long-term postoperative outcomes compared with nonsarcopenic patients. It is thus one consideration to select sublobar resection or stereotactic body radio therapy instead of lobectomy with systematic lymph node dissection, even though it is still unclear whether sublobar resection or stereotactic radiotherapy can improve outcomes of the sarcopenic patients in actual. Second, sarcopenia could be reversible by the combination of nutrition support and exercise therapies in some range $(64,65)$. Therefore, there is a possibility that perioperative nutrition support and intensive rehabilitation therapies lead improved outcomes of sarcopenic patients after pulmonary resection for NSCLC $(66,67)$. Third, it is considerable choice to provide closer follow-up to the poor risk population, but it should be considered case-by-case basis together with other factors including pathologic lymphovascular invasion. Adjuvant chemotherapy for poor risk patients is another choice, even though careful patient selection is further required.

The definition of sarcopenia, especially evaluation method and cut-off value, is not well established to date. Measurement of psoas muscle area is simple and widely used method in evaluating sarcopenia. Some recent studies described that psoas muscle volume $(53,68,69)$ or total skeletal muscle area (70) are more reliable and sensitive despite of the difficulty in measuring. We would also note that lowest quartile of muscle area has been widely used as the cut-off by previous investigators $(13,71-73)$.

There are several limitations in the current study. First, the data were collected and analyzed retrospectively that can cause selection bias. Second, this is single-center study with relatively small sample size. Third, the current measurement method and cut-off value of psoas muscle area are possibly arbitrary even though there is no consensus regarding the cut-off value, measuring methods, and normalization in evaluating sarcopenia (13,71-73). In addition, it should be also noted that some publications demonstrated that contrast media and CT slice thickness can strongly affect the evaluation of skeletal muscle mass (74-76). It may cause methodological errors in evaluating sarcopenia. Although skeletal muscle area on CT imaging is the gold standard of evaluating sarcopenia, it is only an aspect of sarcopenia in 
which includes multiple dimensions such as muscle mass, muscle strength, muscle quality, and physical performance. Recent studies have highlighted decline in the quality and strength of skeletal muscle mass in addition to the quantity (77-79). There are some possibilities that combination of the assessment of skeletal muscle area in addition to physiological and oncological factors could improve capacity to predict outcomes following pulmonary resection for NSCLC. Since skeletal muscle area may differ according to race, sex, and age, it may be difficult to generalize the current findings. We performed analysis with using sexspecific cut-off value, but our cohort consists of single race. That may cause one of the significant limitations.

\section{Conclusions}

In conclusion, it is clinically significant that the sarcopenia group demonstrated unfavorable outcomes in short- and long-term outcomes after surgical resection in only patients with stage I NSCLC. Psoas muscle area measurement is easily applicable as CT imaging is preoperative routine examination in general practice. Similarly, evaluation of sarcopenia may also enable modification of perioperative management, for example, indication of sublobar resection, nutrition support, intensive rehabilitation, follow-up examination, and adjuvant therapy. Further studies on modified management should shed a light on further clinical significance of sarcopenia in patients with earlystage NSCLC.

\section{Acknowledgments}

The authors thank Marissa Mayor, M.D., Department of Surgery, U.S. Naval Hospital Okinawa, for the editorial review of the manuscript.

Funding: None.

\section{Footnote}

Reporting Checklist: The authors have completed the REMARK reporting checklist. Available at http://dx.doi. org/10.21037/atm-20-4380

Data Sharing Statement: Available at http://dx.doi. org/10.21037/atm-20-4380

Peer Review File: Available at http://dx.doi.org/10.21037/ atm-20-4380
Conflicts of Interest: All authors have completed the ICMJE uniform disclosure form (available at http://dx.doi. org/10.21037/atm-20-4380). The authors have no conflicts of interest to declare.

Etbical Statement: The authors are accountable for all aspects of the work in ensuring that questions related to the accuracy or integrity of any part of the work are appropriately investigated and resolved. Given the retrospective study design, the requirement to obtain informed consent was waived. This investigation was approved by the Clinical Research Ethics Committee of Sagamihara Kyodo Hospital (2018-004) and performed in adherence with the Declaration of Helsinki (as revised in 2013).

Open Access Statement: This is an Open Access article distributed in accordance with the Creative Commons Attribution-NonCommercial-NoDerivs 4.0 International License (CC BY-NC-ND 4.0), which permits the noncommercial replication and distribution of the article with the strict proviso that no changes or edits are made and the original work is properly cited (including links to both the formal publication through the relevant DOI and the license). See: https://creativecommons.org/licenses/by-nc-nd/4.0/.

\section{References}

1. Minna JD, Roth JA, Gazdar AF. Focus on lung cancer. Cancer Cell 2002;1:49-52.

2. Ettinger DS, Akerley W, Borghaei H, et al. National Comprehensive Cancer Network. Non-small cell lung cancer, version 2.2013. J Natl Compr Canc Netw 2013;11:645-53.

3. Ginsberg RJ, Rubinstein LV. Randomized trial of lobectomy versus limited resection for T1 N0 non-small cell lung cancer. Lung Cancer Study Group. Ann Thorac Surg 1995;60:615-22.

4. Eguchi T, Bains S, Lee MC, et al. Impact of Increasing Age on Cause-Specific Mortality and Morbidity in Patients with Stage I Non-Small-Cell Lung Cancer: A Competing Risks Analysis. J Clin Oncol 2017;35:281-90.

5. Takahashi Y, Matsuda M, Aoki S, et al. Qualitative Analysis of Preoperative High-Resolution Computed Tomography: Risk Factors for Pulmonary Complications After Major Lung Resection. Ann Thorac Surg 2016;101:1068-74.

6. Sawabata N, Miyaoka E, Asamura H, et al. Japanese Joint Committee for Lung Cancer Registration. Japanese lung 
cancer registry study of 11,663 surgical cases in 2004: demographic and prognosis changes over decade. J Thorac Oncol 2011;6:1229-35.

7. Takahashi Y, Horio H, Hato T, et al. Predictors of postrecurrence survival in patients with non-small-cell lung cancer initially completely resected. Interact Cardiovasc Thorac Surg 2015;21:14-20.

8. Flexman AM, Charest-Morin R, Stobart L, et al. Frailty and postoperative outcomes in patients undergoing surgery for degenerative spine disease. Spine J 2016;16:1315-23.

9. Clegg A, Young J, Iliffe S, et al. Frailty in elderly people. Lancet 2013;381:752-62.

10. Fried LP, Tangen CM, Walston J, et al. Cardiovascular Health Study Collaborative Research Group. Frailty in older adults: evidence for a phenotype. J Gerontol A Biol Sci Med Sci 2001;56:M146-56.

11. Cruz-Jentoft AJ, Bahat G, Bauer J, et al. Sarcopenia: revised European consensus on definition and diagnosis. Age Ageing 2019;48:16-31.

12. Keevil VL, Romero-Ortuno R. Ageing well: a review of sarcopenia and frailty. Proc Nutr Soc 2015;74:337-47.

13. Okamura H, Kimura N, Tanno K, et al. The impact of preoperative sarcopenia, defined based on psoas muscle area, on long-term outcomes of heart valve surgery. J Thorac Cardiovasc Surg 2019;157:1071-1079.e3.

14. Narumi T, Watanabe T, Kadowaki S, et al. Sarcopenia evaluated by fat-free mass index is an important prognostic factor in patients with chronic heart failure. Eur J Intern Med 2015;26:118-22.

15. Hanatani S, Izumiya Y, Onoue Y, et al. Non-invasive testing for sarcopenia predicts future cardiovascular events in patients with chronic kidney disease. Int J Cardiol 2018;268:216-21.

16. Ji Y, Cheng B, Xu Z, et al. Impact of 10sarcopenic obesity on 30-day mortality in critically ill patients with intraabdominal sepsis. J Crit Care 2018;46:50-4.

17. Chakedis J, Spolverato G, Beal EW, et al. Pre-operative Sarcopenia Identifies Patients at Risk for Poor Survival After Resection of Biliary Tract Cancers. J Gastrointest Surg 2018;22:1697-708.

18. Okumura S, Kaido T, Hamaguchi Y, et al. Impact of Skeletal Muscle Mass, Muscle Quality, and Visceral Adiposity on Outcomes Following Resection of Intrahepatic Cholangiocarcinoma. Ann Surg Oncol 2017;24:1037-45.

19. Psutka SP, Carrasco A, Schmit GD. Sarcopenia in patients with bladder cancer undergoing radical cystectomy: impact on cancer-specific and all-cause mortality. Cancer
2014;120:2910-8.

20. Peng P, Moynagh MR, Boorjian SA, et al. Impact of sarcopenia on outcomes following resection of pancreatic adenocarcinoma. J Gastrointest Surg 2012;16:1478-86.

21. Takahashi Y, Eguchi T, Kameda K, et al. Histologic subtyping in pathologic stage I-IIA lung adenocarcinoma provides risk-based stratification for surveillance. Oncotarget 2018;9:35742-51.

22. Takahashi Y, Sakaguchi K, Horio H, et al. Urinary N1, N12-diacetylspermine is a non-invasive marker for the diagnosis and prognosis of non-small-cell lung cancer. Br J Cancer 2015;113:1493-501.

23. Dejima H, Takahashi Y, Hato T, et al. Mediastinal pulmonary artery is associated with greater artery diameter and lingular division volume. Sci Rep 2017;7:1273.

24. Paknikar R, Friedman J, Cron D, et al. Psoas muscle size as a frailty measure for open and transcatheter aortic valve replacement. J Thorac Cardiovasc Surg 2016;151:745-51.

25. National Cancer Institute. Common Terminology Criteria for Adverse Events (CTCAE) Version 4.03. Washington, DC: US Department of Health and Human Services, 2010.

26. National Comprehensive Cancer Network. NCCN clinical practice guidelines in oncology (NSSN Guidelines): Nonsmall cell lung Cancer v5. 2017. Available online: https:// www.nccn.org/patients/guidelines/lung-nsclc/index. html\#68

27. Takahashi Y, Ikeda N, Nakajima J, et al. Prognostic Analysis of Surgical Resection for Pulmonary Metastasis from Hepatocellular Carcinoma. World J Surg 2016;40:2178-85.

28. Shinohara S, Otsuki R, Kobayashi K, et al. Impact of Sarcopenia on Surgical Outcomes in Non-small Cell Lung Cancer. Ann Surg Oncol 2020;27:2427-35.

29. Deng HY, Hou L, Zha P, et al. Sarcopenia is an independent unfavorable prognostic factor of non-small cell lung cancer after surgical resection: A comprehensive systematic review and meta-analysis. Eur J Surg Oncol 2019;45:728-35.

30. Nakada T, Noda Y, Kato D, et al. Risk factors and cancer recurrence associated with postoperative complications after thoracoscopic lobectomy for clinical stage I nonsmall cell lung cancer. Thorac Cancer 2019;10:1945-52.

31. Kawaguchi Y, Hanaoka J, Ohshio Y, et al. Sarcopenia predicts poor postoperative outcome in elderly patients with lung cancer. Gen Thorac Cardiovasc Surg 2019;67:949-54.

32. Nakamura R, Inage $Y$, Tobita R, et al. Sarcopenia in 
Resected NSCLC: Effect on Postoperative Outcomes. J Thorac Oncol 2018;13:895-903.

33. Shi B, Liu S, Chen J, et al. Sarcopenia is Associated with Perioperative Outcomes in Gastric Cancer Patients Undergoing Gastrectomy. Ann Nutr Metab 2019;75:213-22.

34. Zhuang CL, Shen X, Zou HB, et al. EWGSOP2 versus EWGSOP1 for sarcopenia to predict prognosis in patients with gastric cancer after radical gastrectomy: Analysis from a large-scale prospective study. Clin Nutr 2020;39:2301-10.

35. Xu J, Zheng B, Zhang S, et al. Effects of preoperative sarcopenia on postoperative complications of minimally invasive oesophagectomy for oesophageal squamous cell carcinoma. J Thorac Dis 2019;11:2535-45.

36. Jochum SB, Kistner M, Wood EH, et al. Is sarcopenia a better predictor of complications than body mass index? Sarcopenia and surgical outcomes in patients with rectal cancer. Colorectal Dis 2019;21:1372-8.

37. Mayr R, Fritsche HM, Zeman F, et al. Sarcopenia predicts 90-day mortality and postoperative complications after radical cystectomy for bladder cancer. World J Urol 2018;36:1201-7.

38. Silva de Paula N, de Aguiar Bruno K, Azevedo Aredes M, et al. Sarcopenia and Skeletal Muscle Quality as Predictors of Postoperative Complication and Early Mortality in Gynecologic Cancer. Int J Gynecol Cancer 2018;28:412-20.

39. Tsukioka T, Nishiyama N, Izumi N, et al. Sarcopenia is a novel poor prognostic factor in male patients with pathological Stage I non-small cell lung cancer. Jpn J Clin Oncol 2017;47:363-8.

40. Tsukioka T, Izumi N, Kyukwang C, et al. Loss of muscle mass is a novel predictor of postoperative early recurrence in N2-positive non-small-cell lung cancer. Ann Thorac Cardiovasc Surg2018;24:121-6.

41. Shoji F, Matsubara T, Kozuma Y, et al. Relationship between preoperative sarcopenia status and immunonutritional parameters in patients with early-stage nonsmall cell lung cancer. Anticancer Res 2017;37:6997-7003.

42. Hervochon R, Bobbio A, Guinet C, et al. Body mass index and total psoas area affect outcomes in patients undergoing pneumonectomy for cancer. Ann Thorac Surg 2017;103:287-95.

43. Suzuki Y, Okamoto T, Fujishita T, et al. Clinical implications of sarcopenia in patients undergoing complete resection for early non-small cell lung cancer. Lung Cancer 2016;101:92-7.
44. von Haehling S, Morley JE, Anker SD. An overview of sarcopenia: facts and numbers on prevalence and clinical impact. J Cachexia Sarcopenia Muscle 2010;1:129-33.

45. Neto NIP, Murari ASP, Oyama LM, et al. Peritumoural adipose tissue pro-inflammatory cytokines are associated with tumoural growth factors in cancer cachexia patients. J Cachexia Sarcopenia Muscle 2018;9:1101-8.

46. Alves MJ, Figuerêdo RG, Azevedo FF, et al. Adipose tissue fibrosis in human cancer cachexia: the role of TGF pathway. BMC Cancer 2017;17:190.

47. Cavayas YA, Eljaiek R, Rodrigue É, et al. Preoperative Diaphragm Function Is Associated With Postoperative Pulmonary Complications After Cardiac Surgery. Crit Care Med 2019;47:e966-74.

48. Soma D, Kawamura YI, Yamashita S, et al. Sarcopenia, the depletion of muscle mass, an independent predictor of respiratory complications after oncological esophagectomy. Dis Esophagus 2019;32:doy092.

49. Barnes PJ, Celli BR. Systemic manifestations and comorbidities of COPD. Eur Respir J 2009;33:1165-85.

50. Swallow EB, Reyes D, Hopkinson NS, et al. Quadriceps strength predicts mortality in patients with moderate to severe chronic obstructive pulmonary disease. Thorax 2007;62:115-20.

51. Gosker HR, Kubat B, Schaart G, et al. Myopathological features in skeletal muscle of patients with chronic obstructive pulmonary disease. Eur Respir J 2003;22:280-5.

52. Marquis K, Debigaré R, Lacasse Y, et al. Midthigh muscle cross-sectional area is a better predictor of mortality than body mass index in patients with chronic obstructive pulmonary disease. Am J Respir Crit Care Med 2002;166:809-13.

53. Yoshikawa T, Takano M, Miyamoto M, et al. Psoas muscle volume as a predictor of peripheral neurotoxicity induced by primary chemotherapy in ovarian cancers. Cancer Chemother Pharmacol 2017;80:555-61.

54. Chemama S, Bayar MA, Lanoy E, et al. Sarcopenia is Associated with Chemotherapy Toxicity in Patients Undergoing Cytoreductive Surgery with Hyperthermic Intraperitoneal Chemotherapy for Peritoneal Carcinomatosis from Colorectal Cancer. Ann Surg Oncol 2016;23:3891-8.

55. Wilson D, Jackson T, Sapey E, et al. Frailty and sarcopenia: The potential role of an aged immune system. Ageing Res Rev 2017;36:1-10.

56. Pinke KH, Calzavara B, Faria PF, et al Proinflammatory profile of in vitro monocytes in the ageing is affected by lymphocytes presence. Immun Ageing 2013;10:22. 
57. Doyle KP, Cekanaviciute E, Mamer LE, et al. TGF $\beta$ signaling in the brain increases with aging and signals to astrocytes and innate immune cells in the weeks after stroke. J Neuroinflammation 2010;7:62.

58. Duggal NA, Upton J, Phillips AC, et al. An age-related numerical and functional deficit in CD19(+) CD24(hi) CD38(hi) B cells is associated with an increase in systemic autoimmunity. Aging Cell 2013;12:873-81.

59. Lutz CT, Quinn LS. Sarcopenia, obesity, and natural killer cell immune senescence in aging: altered cytokine levels as a common mechanism. Aging (Albany NY) 2012;4:535-46.

60. Coppé JP, Desprez PY, Krtolica A, et al. The senescenceassociated secretory phenotype: the dark side of tumor suppression. Annu Rev Pathol 2010;5:99-118.

61. Crossland H, Constantin-Teodosiu D, Gardiner SM, et al. A potential role for Akt/FOXO signalling in both protein loss and the impairment of muscle carbohydrate oxidation during sepsis in rodent skeletal muscle. J Physiol 2008;586:5589-600.

62. Pedersen BK, Febbraio MA. Muscle as an endocrine organ: focus on muscle-derived interleukin-6. Physiol Rev 2008;88:1379-406.

63. Shubert TE. Evidence-based exercise prescription for balance and falls prevention: a current review of the literature. J Geriatr Phys Ther 2011;34:100-8.

64. Aredes MA, da Camara AO, de Paula NS, et al. Efficacy of $\omega-3$ supplementation on nutritional status, skeletal muscle, and chemoradiotherapy toxicity in cervical cancer patients: A randomized, triple-blind, clinical trial conducted in a middle-income country. Nutrition 2019;67-68:110528.

65. Gioia S, Merli M, Nardelli S, et al. The modification of quantity and quality of muscle mass improves the cognitive impairment after TIPS. Liver Int 2019;39:871-7.

66. Saito H, Hatakeyama K, Konno H, et al. Impact of pulmonary rehabilitation on postoperative complications in patients with lung cancer and chronic obstructive pulmonary disease. Thorac Cancer 2017;8:451-60.

67. Mimae T, Suzuki K, Tsuboi M, et al. Severity of lung fibrosis affects early surgical outcomes of lung cancer among patients with combined pulmonary fibrosis and emphysema. Medicine (Baltimore) 2016;95:e4314.

68. Rueth NM, Parsons HM, Habermann EB, et al. Surgical treatment of lung cancer: predicting postoperative morbidity in the elderly population. J Thorac Cardiovasc Surg 2012;143:1314-23.

69. Noguchi G, Kawahara T, Kobayashi K, et al. A lower psoas muscle volume was associated with a higher rate of recurrence in male clear cell renal cell carcinoma. PLoS
One 2020;15:e0226581.

70. Park J, Gil JR, Shin Y, Won SE, et al. Reliable and robust method for abdominal muscle mass quantification using CT/MRI: An explorative study in healthy subjects. PLoS One 2019; 14:e0222042.

71. Fujikawa H, Araki T, Okita Y, et al. Impact of sarcopenia on surgical site infection after restorative proctocolectomy for ulcerative colitis. Surg Today 2017;47:92-8.

72. Amini N, Spolverato G, Gupta R, et al. Impact Total Psoas Volume on Short- and Long-Term Outcomes in Patients Undergoing Curative Resection for Pancreatic Adenocarcinoma: a New Tool to Assess Sarcopenia. J Gastrointest Surg 2015;19:1593-602.

73. Ganapathi AM, Englum BR, Hanna JM, et al. Frailty and risk in proximal aortic surgery. J Thorac Cardiovasc Surg 2014;147:186-191.e1.

74. Morsbach F, Zhang YH, Martin L, et al. Body composition evaluation with computed tomography: Contrast media and slice thickness cause methodological errors. Nutrition 2019;59:50-5.

75. Montano-Loza AJ, Angulo P, Meza-Junco J, et al. Sarcopenic obesity and myosteatosis are associated with higher mortality in patients with cirrhosis. J Cachexia Sarcopenia Muscle 2016;7:126-35.

76. Mourtzakis M, Prado CM, Lieffers JR, et al. A practical and precise approach to quantification of body composition in cancer patients using computed tomography images acquired during routine care. Appl Physiol Nutr Metab 2008;33:997-1006.

77. Kiriya Y, Toshiaki N, Shibasaki I, et al. Sarcopenia assessed by the quantity and quality of skeletal muscle is a prognostic factor for patients undergoing cardiac surgery. Surg Today 2020;50:895-904.

78. Barbalho ER, Gonzalez MC, Bielemann RM, et al. Is skeletal muscle radiodensity able to indicate physical function impairment in older adults with gastrointestinal cancer? Exp Gerontol 2019;125:110688.

79. Wilkinson TJ, Gould DW, Nixon DGD, et al. Quality over quantity? Association of skeletal muscle myosteatosis and myofibrosis on physical function in chronic kidney disease. Nephrol Dial Transplant 2019;34:1344-53.

Cite this article as: Takahashi Y, Suzuki S, Hamada K, Nakada T, Oya Y, Sakakura N, Matsushita H, Kuroda H. Sarcopenia is poor risk for unfavorable short- and long-term outcomes in stage I non-small cell lung cancer. Ann Transl Med 2021;9(4):325. doi: 10.21037/atm-20-4380 


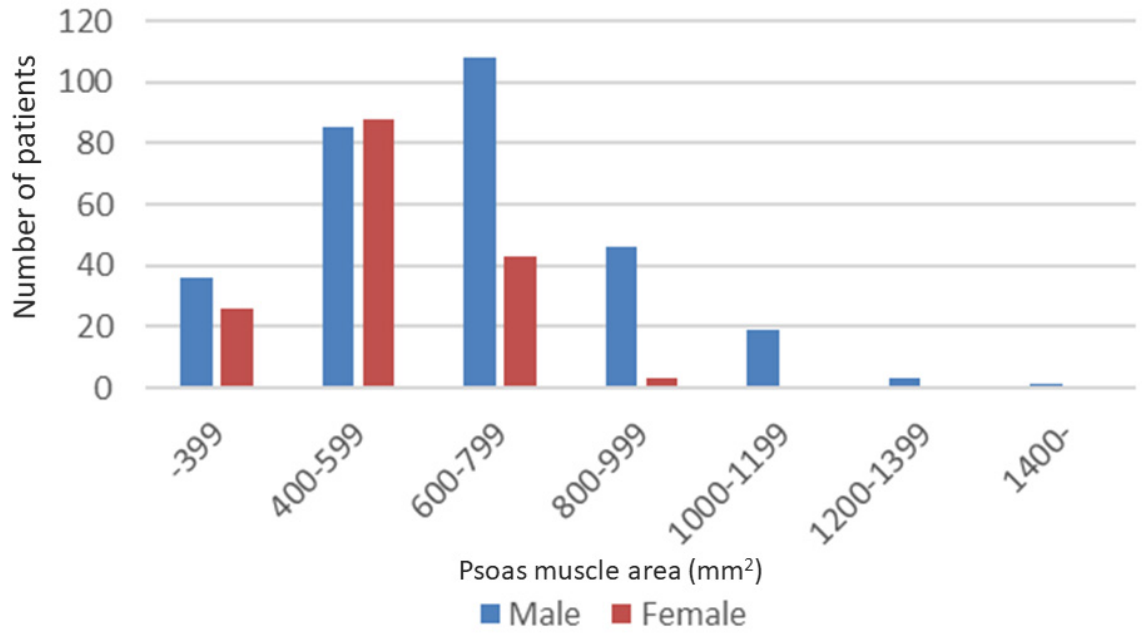

Figure S1 A bar graph that demonstrates distribution of psoas muscle area according to sex.

Table S1 Frequency of postoperative severe complications

\begin{tabular}{lc}
\hline Complications & N \\
\hline Acute respiratory distress syndrome & 3 \\
Pneumonia & 14 \\
Pulmonary fistula & 41 \\
Bronchopleural fistula & 3 \\
Pleural effusion & 3 \\
Atelectasis & 15 \\
Atrial fibrillation & 18 \\
Cerebral infarction & 4 \\
Myocardial infarction & 2 \\
Pleural infection & 4 \\
Chylothorax & 8 \\
Delirium & 2 \\
Heart failure & 1 \\
Acute exacerbation of interstitial & 2 \\
pneumonitis & 4 \\
Wound infection &
\end{tabular}


Table S2 Multivariable logistic regression analysis of recurrence-free survival

\begin{tabular}{|c|c|c|c|c|c|c|}
\hline Variables & \multicolumn{3}{|c|}{ Univariable analysis } & \multicolumn{3}{|c|}{ Multivariable analysis } \\
\hline Body mass index $<18.5$ (vs. $\geq 18.5$ ) & 1.542 & $0.654-2.674$ & 0.194 & $\mathrm{~N} / \mathrm{A}$ & - & - \\
\hline Age $\geq 70$ y/o (vs. $<70$ y/o) & 1.593 & $1.051-2.415$ & 0.022 & 1.594 & $1.065-2.319$ & 0.034 \\
\hline Male (vs. female) & 1.542 & $1.009-2.325$ & 0.043 & 1.361 & $0.897-2.212$ & 0.119 \\
\hline Diabetes (vs. non-diabetes) & 1.368 & $0.694-2.168$ & 0.282 & N/A & - & - \\
\hline $\begin{array}{l}\text { Serum CEA } \geq 5.0 \mathrm{ng} / \mathrm{mL} \\
\text { (vs. }<5.0 \mathrm{ng} / \mathrm{mL} \text { ) }\end{array}$ & 1.034 & $1.021-1.049$ & $<0.001$ & 1.037 & $1.021-1.056$ & $<0.001$ \\
\hline$\% F V C<80.0$ (vs. $\geq 80.0)$ & 1.043 & $0.467-2.428$ & 0.814 & $\mathrm{~N} / \mathrm{A}$ & - & - \\
\hline $\begin{array}{l}\text { Non-adenocarcinoma } \\
\text { (vs. adenocarcinoma) }\end{array}$ & 1.512 & $0.897-1.983$ & 0.112 & $\mathrm{~N} / \mathrm{A}$ & - & - \\
\hline Pathologic stage IB (vs. IA) & 1.386 & $0.906-2.092$ & 0.124 & N/A & - & - \\
\hline
\end{tabular}

*, Cox-regression analysis was used. Cl, confidence interval; CEA, carcinoembryonic antigen; FEV1, forced expiratory volume in 1 second;

FVC, forced vital capacity. 